\title{
ALK-positive anaplastic large cell lymphoma undiagnosed in a patient with tuberculosis: a case report and review of the literature
}

\author{
Weerapat Owattanapanich ${ }^{1 *}$, Pakpoom Phoompoung ${ }^{2}$ and Sanya Sukpanichnant ${ }^{3}$
}

\begin{abstract}
Background: Due to a similarity between the features of lymphoma and the features of tuberculosis, lymphoma may go unrecognized and undiagnosed in patients with tuberculosis.

Case presentation: A 33-year-old Thai man presented to our center with typical clinical manifestations of tuberculous lymphadenitis, with negative tests for both acid-fast bacilli and fungi, and negative polymerase chain reaction for Mycobacterial tuberculosis complex. The disease was not responding to anti-tuberculosis treatment and he developed both pericardial effusion and progressive lymphadenopathy. Large lymphoma cells were evident in the pericardial effusion, and a review of the previous lymph node biopsies confirmed the existence of ALK-positive anaplastic large cell lymphoma and tuberculous lymphadenitis. Moreover, when the tests were repeated, he was found to be positive for both acid-fast bacilli and Mycobacterial tuberculosis complex. The presence of typical morphology of tuberculous lymphadenitis and inattentional blindness may explain why the presence of large lymphoma cells was overlooked in one of the previous lymph node biopsies. Our patient developed severe pneumonia with profound septic shock due to carbapenem-resistant Enterobacteriaceae and died within days.

Conclusions: Given that tuberculosis and lymphoma can share common features, this case highlights the importance of thoroughly reviewing all foregoing relevant patient data (most notably pathology samples) in order to rule out the presence of lymphoma that may exist within the shadow of typical morphology of tuberculous lymphadenitis.
\end{abstract}

Keywords: ALK protein, Anaplastic large cell lymphoma, Tuberculosis, Undiagnosed lymphoma, Inattentional blindness

\section{Background}

Tuberculosis (TB) is an infectious disease that causes significant morbidity and mortality, and is one of the top ten causes of death worldwide. In 2015 alone, more than 10 million people became infected with $\mathrm{TB}$ and 1.8 million died from the disease. Over 95\% of TB-related deaths occur in low-income or middle-income countries. Due to advancements in care for patients with TB, an estimated 49 million lives were saved between 2000 and 2015. However, the number of people with multidrug-resistant TB (MDR-TB) was reported to be an estimated 480,000

\footnotetext{
*Correspondence: weerapato36733@gmail.com

'Division of Hematology, Department of Medicine, Faculty of Medicine Siriraj Hospital, Mahidol University, Bangkok, Thailand

Full list of author information is available at the end of the article
}

globally in 2015. When a patient with TB fails to respond to anti-TB therapy, potential causes other than MDR-TB must also be considered, including drug compliance, inadequate care, relapse, and superimposed human immunodeficiency virus (HIV) infection, among others [1]. Another possible cause of unresponsiveness to anti-TB therapy is undiagnosed malignancy (especially malignant lymphoma) in which constitutional symptoms and lymphadenopathy still persist after TB therapy has commenced [2]. Here, we report a case of undiagnosed ALK-positive anaplastic large cell lymphoma (ALK+ ALCL) in a patient with TB to highlight the importance of reviewing all foregoing related patient data (especially pathological samples), and the relative difficulty associated with reaching definitive diagnosis in patients with these coexisting conditions. 


\section{Case presentation}

A 33-year-old Thai man presented at our center with a mass on the left side of his neck for 3 weeks. Our center, Siriraj Hospital (Bangkok, Thailand), is Thailand's largest national tertiary referral center. Three years earlier, he sought treatment at a private hospital for a low-grade fever and a palpable mass at his chest wall. A physical examination revealed generalized lymphadenopathy, including left posterior cervical, supraclavicular, and suprasternal lymph nodes that varied from 1 to $2 \mathrm{~cm}$ in diameter. Aspiration of the suprasternal mass was performed and pus culture was positive for Pseudomonas aeruginosa. He was prescribed orally administered sitafloxacin $100 \mathrm{mg}$ once daily for 1 week. After being treated, the masses significantly decreased in size to the point where they were no longer palpable, and he became afebrile with good appetite.

He re-presented to the same hospital 30 months later with a recurrent suprasternal mass. A biopsy of the mass was performed and the tissue sample was submitted to a private pathology laboratory. The pathologic diagnosis was caseating granuloma, but negative for acid-fast bacilli (AFB) and fungi. Polymerase chain reaction (PCR) for Mycobacterial tuberculosis complex using deoxyribonucleic acid (DNA) extract from the tissue block was reported as negative. Empirical antibiotics were prescribed and a transient clinical response was observed.

Five months later, he developed a matted left cervical lymph node that measured $3 \mathrm{~cm}$ in diameter that was accompanied by a low-grade fever.

He then went to another hospital where he underwent excisional biopsy of the enlarged cervical lymph node. That tissue sample was submitted to a different private pathology laboratory. The pathologic diagnosis was granulomatous lymphadenitis suggestive of TB, but negative for AFB. Due to the high prevalence of TB in Thailand, the physicians at that hospital decided to treat the patient according to a diagnosis of tuberculous lymphadenitis. PCR for Mycobacterial tuberculosis complex using DNA extract from the tissue block was reported as negative. Six days after anti-TB therapy was started, his cervical lymph nodes increased in size. Computed tomography (CT) of his chest revealed the following: (1) an ill-defined enhancing mass measuring $3.6 \times 2.4 \mathrm{~cm}$ at the left anterolateral oropharynx; (2) a matted cervical lymph node measuring $1.2 \times 1.9 \mathrm{~cm}$; and, (3) multiple mediastinal lymph nodes varying from 0.7 to $1.8 \mathrm{~cm}$ in diameter, with extension to the hilum of his left lung and causing atelectasis of the anterior segment of the upper lobe of his left lung, a small amount of left pleural effusion, and pericardial effusion.

At this point in the evolution of this case, the patient was referred to our center for further investigation and treatment. He and his family worked in their own grocery store and he denied any exposure to TB. At our center, a physical examination revealed the following findings: (1) a matted left cervical lymph node $(3 \mathrm{~cm}$ in diameter), with signs of inflammation; (2) right supraclavicular lymph node (3 $\mathrm{cm}$ in diameter); and, (3) a left tonsillar mass $(2 \mathrm{~cm}$ in diameter). He had a low-grade fever, but no pallor, jaundice, or hepatosplenomegaly was detected. Investigations included blood tests, pericardiocentesis, and a review of pathologic specimens of suprasternal and cervical lymph nodes that were harvested at previous hospitals. A complete blood count showed hemoglobin $14.6 \mathrm{~g} / \mathrm{dL}$, hematocrit $46.4 \%$, white blood cells $(\mathrm{WBC}) 12.59 \times 10^{9} / \mathrm{L}$ with neutrophil predominance, and platelet count $469 \times 10^{9} / \mathrm{L}$. His serum lactate dehydrogenase (LDH) level was $534 \mathrm{U} /$ L. Anti-HIV was negative. Pericardial fluid profiles showed straw-colored fluid; protein $4.81 \mathrm{~g} / \mathrm{dl}$; LDH $2292 \mathrm{U} / \mathrm{L}$; and, cell count $166 \times 10^{9} / \mathrm{L}$ with abnormal large cells $89 \%$, consistent with large lymphoma cells (Fig. 1). Special stain was negative for AFB, and PCR for $\mathrm{TB}$ was negative. A bone marrow examination was negative for malignant lymphoma.

A pathologic review of the two previous lymph node biopsies was performed. The first biopsy was an excision of an enlarged suprasternal lymph node that showed typical features of tuberculous lymphadenitis, with multiple granulomas with caseous necrosis and some Langhans giant cells. The remaining lymph node tissue that was unaffected by granulomatous reaction showed occasional activated lymphoid cells within a background of small lymphoid cells. Few AFB were identified (Fig. 2). PCR for Mycobacterium tuberculosis complex was performed using DNA extract from lymph node tissue left in the paraffin block, and the result was positive for Mycobacterium

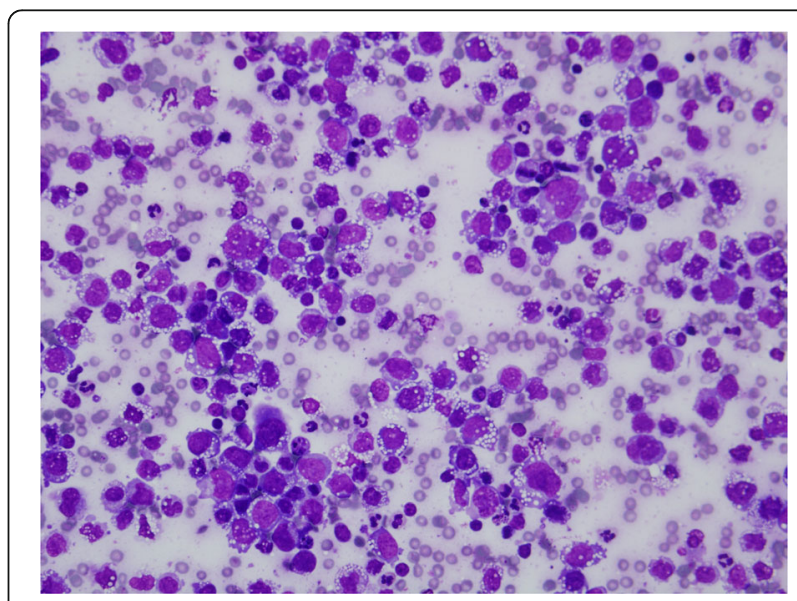

Fig. 1 Large lymphoma cells in the pericardial effusion. Lymphoma cells vary in size, from 5 to 15 times that of normal erythrocytes. Nuclei are pleomorphic, with some coarse nuclear chromatins and distinct nucleoli. Cytoplasm is faint blue to basophilic with frequent vacuoles (Diff-Quik ${ }^{\oplus}$, 1000X) 
tuberculosis complex. Thus, a definite diagnosis of TB was established.

In contrast, the second biopsy of his left cervical lymph node was an incisional biopsy and it was received in multiple tissue fragments. Only one small area of the biopsy showed a few fibrocaseous granulomas, but most of the tissue showed irregular pink areas that resembled necrotic areas when observed at low magnification $(\times 2$ or $\times 4)$. However, under high magnification (at least $\times 40$ ), these pink areas contained a large number of abnormal large cells. Immunohistochemistry showed that these large cells were positive for CD30, ALK, CD4, and TIA-1, and the proliferation index by Ki-67 was high $(>90 \%)$. However, these cells were negative for CD3, CD5, CD10, CD20, CD68, CyclinD1, and BCL2. Special stains were negative for AFB and fungi. In situ hybridization for Epstein-Barr virus-encoded small ribonucleic acid (EBER) study was negative. Given the pathological findings of this second lymph node biopsy, a diagnosis of ALK+ ALCL with focal granulomatous reaction and focal necrosis was made (Fig. 3). Our patient received anti-TB drugs, including isoniazid, rifampicin, pyrazinamide, and ethambutol, combined with cyclophosphamide, hydroxydaunorubicin, Oncovin (vincristine), etoposide, and prednisone (CHOEP) regimen every 3 weeks for six cycles as a standard chemotherapy for ALK+ ALCL. He had no adverse events or drug interaction from either anti-TB drugs or chemotherapy. Although this lymphoma subtype was expected to respond well to chemotherapy, he achieved only a stable response, which is defined as a decrease in node size less than $50 \%$ compared to initial node size at the end of the course of treatment. Lymphoma subtypes that express CD30 are expected to respond well to brentuximab vedotin, which is an anti-CD30 therapy; however, this drug has not been approved for use in Thailand. As an alternative, ifosfamide, carboplatin, and etoposide (ICE) regimen, which is a salvage combination chemotherapy, was given. Our patient then developed severe pneumonia with profound septic shock due to carbapenem-resistant Enterobacteriaceae and died a few days later. His family did not grant permission for a postmortem examination.

In order to determine any minimal involvement of lymphoma in the first lymph node biopsy and for bone marrow staging, immunostaining was performed for CD30 and ALK protein in both samples. In the first lymph node biopsy, some of the scattered large activated lymphoid cells showed CD30 positivity, but none showed ALK protein expression. In the bone marrow, no CD30+ or ALK+ cells were detected.

\section{Discussion}

After initially testing negative for AFB and Mycobacterium tuberculosis complex, the patient in this case report was later definitively diagnosed as having tuberculous lymphadenitis by typical pathologic features, identification of AFB, and positive PCR for Mycobacterium tuberculosis complex. Similarly and slightly more than 5 months later, he was diagnosed as having large lymphoma cells in the pericardial effusion, after no sign of lymphoma cells was detected in two previous lymph node samples. The second

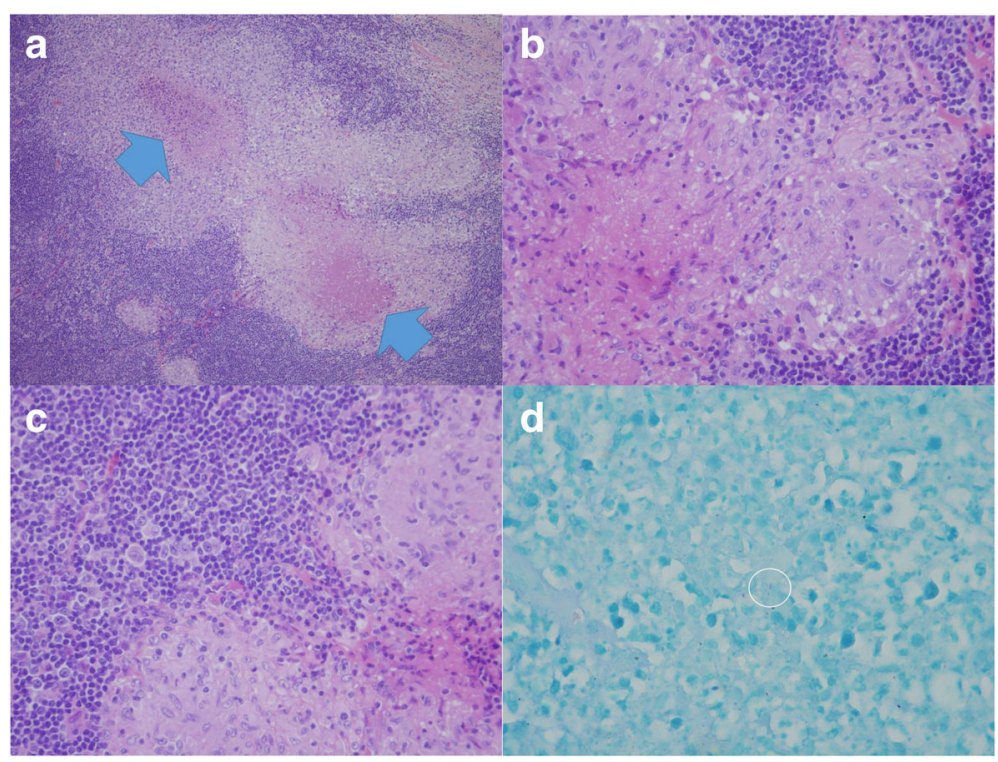

Fig. 2 Review of the first lymph node biopsy revealed: a Typical caseating granulomas (arrows) at low magnification (hematoxylin and eosin stain, $\times 10$ ); $\mathbf{b}$ typical epithelioid histiocytes in granulomas at high magnification (hematoxylin and eosin stain, $\times 1000$ ); $\mathbf{c}$ some scattered activated lymphoid cells outside the granuloma proven to be reactive (hematoxylin and eosin stain, $\times 1000$ ); and, $\mathbf{d}$ detection of acid-fast bacillus within the white circle (hematoxylin and eosin stain, $\times 1000)$ 


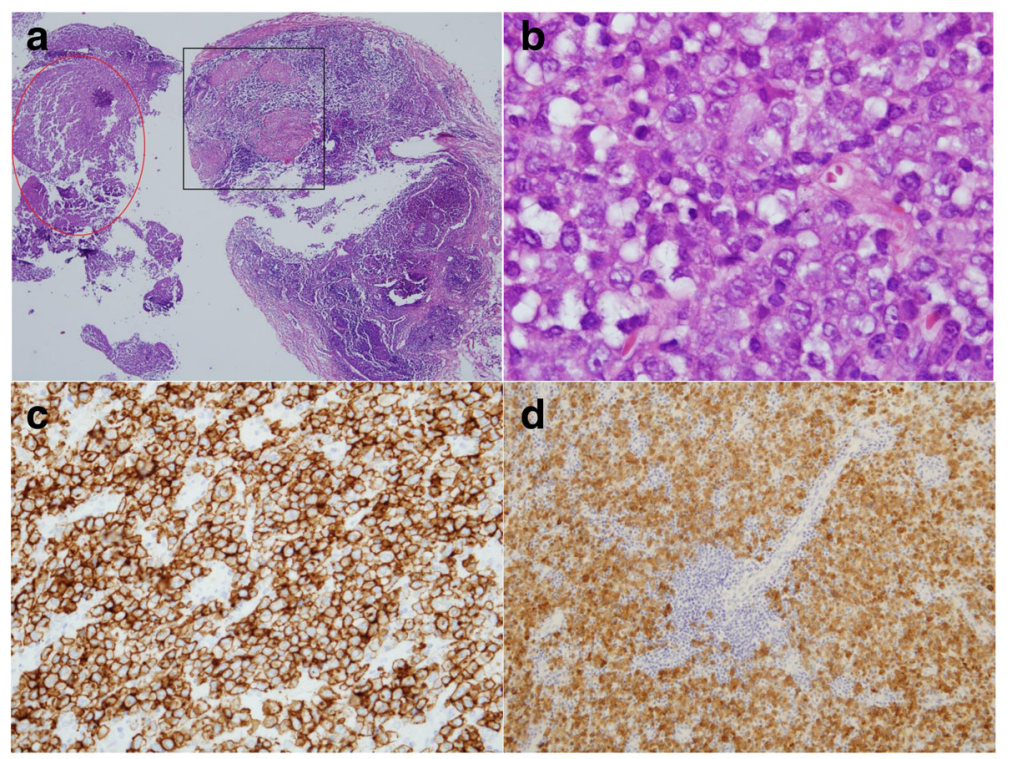

Fig. 3 Review of the second lymph node biopsy revealed: a Typical fibrocaseous granulomas in black rectangular box and lymphoma area in red oval at low magnification (hematoxylin and eosin stain, $\times 4$ ); b large lymphoma cells at high magnification (hematoxylin and eosin stain, $\times 1000$ ); $\mathbf{c}$ CD30+ lymphoma cells (immunostained for CD30, x40); and, $\mathbf{d}$ ALK + lymphoma cells (immunostained for ALK protein, 20x)

lymph node biopsy was reviewed and undiagnosed ALK+ ALCL was discovered. This may have been due to irregular pink areas of large lymphoma cells that were overlooked by the pathologist who originally made the diagnosis of "granulomatous lymphadenitis, suggestive of TB, but negative for AFB." It is possible that these pink lymphomatous areas were misinterpreted at low magnification as necrotic areas that are commonly seen in tuberculous lymphadenitis. If these areas had been examined under high magnification, large lymphoma cells would have been identified and a diagnosis of accompanying malignant lymphoma could have been made. A phenomenon known as "inattentional blindness" or "perceptual blindness" may also explain why large lymphoma cells were not observed during the search for AFB at high magnification of the second lymph node biopsy [3]. Inattentional blindness is defined as a failure to observe obvious events or stimuli that are peripheral to what you are seeking to find.

Malignant lymphoma could go undiagnosed or be misdiagnosed as TB [2], because lymphoma cells can induce granulomatous reaction [4-6]. The exact mechanism of granulomatous reaction is not known, but it is believed that lymphoma cells may produce some cell products or cytokines that directly induce granulomatous reaction or that indirectly activate immune cells involved in the formation of granulomatous reaction. In our review of the literature, we found only one case of coexisting ALK + ALCL and pulmonary TB [7]. That patient had excellent outcomes from treatment of both TB infection and lymphoma. In contrast, our patient had a refractory response to both standard and salvage chemotherapy.
Anaplastic large cell lymphoma (ALCL) was introduced as a unique variety of non-Hodgkin lymphoma in the 2001 version of the World Health Organization (WHO) classification [8]; ALK+ ALCL was later listed in the 2008 version of the WHO classification [9]; and, ALK-negative ALCL was recently added in the 2016 version of the WHO classification [10]. According to the 2008 version of the WHO classification, ALK+ ALCL is a rare type of mature T cell neoplasm. Morphology of the lymphoma cell is large-sized with abundant cytoplasm and pleomorphic, horseshoe-shaped, nuclei. The age range of patients is from 10 to 30 years, with a male predominance (male to female ratio of 1.5:1). B symptoms, especially fever, usually develop as the initial manifestation. Common sites of lymphoma involvement include lymph node, skin, bone, soft tissue, lungs, and liver. Compared to other $\mathrm{T}$ cell lymphoma subtypes, ALK+ ALCL has a better prognosis, with a 5-year failure-free survival (FFS) rate of $60 \%[9,11]$. However, the patient profiled in this report had a grave clinical outcome, which was probably due to the advanced stage of his disease.

Pulmonary TB has been shown to increase the risk of lung cancer development [12]. However, TB has not been shown to associate with malignant lymphoma, except for pyothorax-associated lymphoma, which is a rare Epstein-Barr virus (EBV)-associated lymphoma within the inflammatory cavity that has been reported in patients with TB-associated pyothorax [13]. In the case presented here, coexisting tuberculous lymphadenitis and ALK+ ALCL appears to be merely coincidental, because no association between the two diseases has been 
reported, and ALK+ ALCL in our patient was not associated with EBV infection. It is also not possible to exclude the role of chronic infection in $\mathrm{TB}$ as a predisposing factor in lymphomagenesis. Hypothetically, TB infection contributes to increased reactive oxygen species (ROS), which results in DNA damage, elevated anti-apoptotic activity from enhancing BCL2 synthesis, and increased levels of several cytokines that relate to angiogenesis, such as leukotrienes, prostaglandins, and vascular endothelial growth factor (VEGF) [14].

If a diagnosis of TB had not been established in this case, the large lymphoma cells found in the pericardial effusion and in the second lymph node biopsy would have been strong evidence of ALK+ ALCL, and the granulomas found in both lymph nodes may have been interpreted as a reaction to lymphoma cells. It is, however, very unusual to observe such obvious caseous necrosis in granulomatous reaction in malignant lymphoma that is usually described as non-necrotic [4-6]. Further investigation would, therefore, be required to identify possible mycobacterium species and/or other causative pathogens in order to identify the cause of the caseating granulomas. In this case, upon the review, careful searching for AFB yielded a positive result, which then led to repeat PCR for Mycobacterium tuberculosis complex using DNA extract from the first lymph node biopsy that also turned out to be positive. The fact that the first tissue biopsy report showed positive for AFB with no lymphoma cells being observed is an indication that lymphoma cells may only be found in a certain portion of lymph node. Accordingly, excisional or core biopsy should be emphasized instead of fine-needle aspiration (FNA), especially if lymphoma is a differential diagnosis.

\section{Conclusions}

Here, we presented a case of undiagnosed ALK+ ALCL in a patient with TB. Given that TB and lymphoma can share common features, this case highlights the importance of thoroughly reviewing all foregoing germane patient data (most notably pathology samples) in order to rule out the presence of lymphoma that may exist within the shadow of typical morphology of tuberculous lymphadenitis.

\begin{abstract}
Abbreviations
AFB: Acid-fast bacilli; ALCL: Anaplastic large cell lymphoma; ALK+ ALCL: ALK-positive anaplastic large cell lymphoma; CHOEP: Cyclophosphamide, hydroxydaunorubicin, Oncovin (vincristine), etoposide, and prednisone; CT: Computed tomography; EBER: Epstein-Barr virus-encoded small RNA; EBV: Epstein-Barr virus; FFS: Failure-free survival; FNA: Fine-needle aspiration; ICE: Ifosfamide, carboplatin, and etoposide; LDH: Lactate dehydrogenase; MDR-TB: Multidrug-resistant tuberculosis; PCR: Polymerase chain reaction; ROS: Reactive oxygen species; TB: Tuberculosis; VEGF: Vascular endothelial growth factor; WBC: White blood cells; WHO: World Health Organization
\end{abstract}

\section{Acknowledgements}

The authors gratefully acknowledge the patient profiled in this report for permitting us to publish details about his case.
Funding

This was an unfunded study.

Availability of data and materials

Data sharing is not applicable to this article, as no datasets were generated or analyzed.

\section{Authors' contributions}

WO and SS analyzed and interpreted patient data and drafted the manuscript. SS performed the histological examination, made the diagnosis, and created the figures. WO and PP performed physical examinations and provided medical care. All authors read and approved the final manuscript.

\section{Competing interests}

The authors declare that they have no competing interests.

\section{Consent for publication}

Written informed consent was obtained from the patient for publication of this case report and the accompanying images. A copy of the written consent is available for review by the Editor-in-Chief of this journal.

Ethical approval and consent to participate

No ethics committee approval is required at our institution for a case report involving a single patient.

\section{Publisher's Note}

Springer Nature remains neutral with regard to jurisdictional claims in published maps and institutional affiliations.

\section{Author details}

${ }^{1}$ Division of Hematology, Department of Medicine, Faculty of Medicine Siriraj Hospital, Mahidol University, Bangkok, Thailand. ²Division of Infectious

Diseases and Tropical Medicine, Department of Medicine, Faculty of Medicine Siriraj Hospital, Mahidol University, Bangkok, Thailand. ${ }^{3}$ Department of Pathology, Faculty of Medicine Siriraj Hospital, Mahidol University, Bangkok, Thailand.

Received: 22 November 2016 Accepted: 7 April 2017

Published online: 11 May 2017

\section{References}

1. WHO. Global tuberculosis report 2016. Geneva: WHO; 2016. http://www. who.int/tb/publications/global_report/en/index.html. Accessed 1 Nov 2016.

2. Puvaneswaran B, Shoba B. Misdiagnosis of tuberculosis in patients with lymphoma. S Afr Med J. 2012;103(1):32-3.

3. Mack A, Tang B, Tuma R, Kahn S, Rock I. Perceptual organization and attention. Cogn Psychol. 1992;24(4):475-501.

4. Braylan RC, Long JC, Jaffe ES, Greco FA, Orr SL, Berard CW. Malignant lymphoma obscured by concomitant extensive epithelioid granulomas: report of three cases with similar clinicopathologic features. Cancer. 1977;39:1146-55.

5. Sacks EL, Donaldson SS, Gordon J, Dorfman RF. Epithelioid granulomas associated with Hodgkin's disease: clinical correlations in 55 previously untreated patients. Cancer. 1978:41:562-7.

6. Haralambieva E, Rosati S, van Noesel C, Boers E, van Marwijk KM, Schuuring E, et al. Florid granulomatous reaction in Epstein-Barr virus-positive nonendemic Burkitt lymphomas: report of four cases. Am J Surg Pathol. 2004;28(3):379-83.

7. Baka M, Doganis D, Pourtsidis A, Tsolia M, Bouhoutsou D, Varvoutsi M, et al. Successful treatment in a child with anaplastic large cell lymphoma and coexistence of pulmonary tuberculosis. Case Rep Pediatr. 2013;2013:928701. doi:10.1155/2013/928701. Epub 2013 Jun 13.

8. Jaffe ES, Harris NL, Stein H, Vardiman JW. World Health Organization classification of tumors. Pathology and genetics of tumours of haematopoietic and lymphoid tissues. Lyon: IARC Press; 2001.

9. Swerdlow SH, Campo E, Harris NL, et al. World Health Organization classification of tumors. Pathology and genetics of tumours of haematopoietic and lymphoid tissues. Lyon: IARC Press; 2008.

10. Swerdlow SH, Campo E, Pileri SA, et al. The 2016 revision of the World Health Organization classification of lymphoid neoplasms. Blood. 2016;127(20):2375-90 
11. Kinney MC, Higgins RA, Medina EA. Anaplastic large cell lymphoma: twenty-five years of discovery. Arch Pathol Lab Med. 2011;135(1):19-43.

12. Wu CY, Hu HY, Pu CY, Huang N, Shen HC, Li CP, Chou YJ. Pulmonary tuberculosis increases the risk of lung cancer: a population-based cohort study. Cancer. 2011;117(3):618-24

13. Fukayama M, Hayashi Y, Ooba T, et al. Pyothorax-associated lymphoma: development of Epstein-Barr virus-associated lymphoma within the inflammatory cavity. Pathol Int. 1995;45(11):825-31.

14. Falagas ME, Kouranos VD, Athanassa Z, Kopterides P. Tuberculosis and malignancy. QJM. 2010;103(7):461-87.

Submit your next manuscript to BioMed Central and we will help you at every step:

- We accept pre-submission inquiries

- Our selector tool helps you to find the most relevant journal

- We provide round the clock customer support

- Convenient online submission

- Thorough peer review

- Inclusion in PubMed and all major indexing services

- Maximum visibility for your research

Submit your manuscript at www.biomedcentral.com/submit
Biomed Central 